\title{
Skaphoidpseudarthrosen - wenn das Kahnbein nicht heilen will
}

Simon Oeckenpöhler, Johanna Ueberberg, Michael J. Raschke, Martin F. Langer

Skaphoidpseudarthrosen führen häufig zum karpalen Kollaps mit Ausbildung einer schweren posttraumatischen Arthrose und dadurch zu massiven Einschränkungen des Handgelenkes. Dieser Artikel soll den Weg von der Fraktur zur Pseudarthrose sowie aktuelle Diagnostikund Therapiekonzepte vorstellen und beleuchten.

Brüche des Kahnbeins treten mit einer Inzidenz von 10/ 100000 Lebensjahren im Alter bis 65 Jahren auf [1] und übertreffen so mit einem Anteil von ca. $80 \%$ aller Frakturen des Karpus mit Abstand sämtliche anderen Entitäten der Karpusverletzungen.

\section{Anatomische Besonderheiten - Knorpel, Durchblutung und Biomechanik}

Das Kahnbein ist in der Handwurzel proximal radial zwischen Speiche proximal und kleinem und großem Vieleckbein distal gelegen und wird nach ulnar von Mondund Kopfbein eingeschlossen. Diese vielen Kontaktflächen sind gelenkbildend, sodass das Skaphoid (je nach Quellenangabe) zu 42-80\% mit Knorpel überzogen ist $[2,3]$.

Die Durchblutung erfolgt über die A. radialis, wobei der größere proximale Anteil des Knochens (70-80\%) von dorsal (R. dorsalis scaphoidei) versorgt wird. Dieser läuft im dorsalen Sulcus scaphoidei. Von palmar erfolgt die Blutversorgung über den Arcus intercarpalis palmaris [3] und versorgt so die übrigen $20-30 \%$ und damit den distalen Anteil ( $\triangleright$ Abb. 1).

Dieses System ist vor allem dorsal störungsanfällig und muss in jedweder operativer Intervention nach Möglichkeit geschont werden, um keine Nekrose zu riskieren. Der retrograden Versorgung des proximalen Pols kommt hier eine besondere Bedeutung zu, da diese bei Frakturen mit einer deutlich erhöhten Rate an avaskulären Nekrosen des proximalen Pols und damit Ausbildung einer Pseudarthrose assoziiert ist.

Als zweitgrößter Knochen im Handgelenk spielt das Scaphoid eine wichtige Rolle in der Kinematik des Karpus. Lichtman beschrieb einen karpalen „Ring“, in dem die Handwurzelknochen mit und gegeneinander verspannt sind. In der 1. Reihe ist das Kahnbein mit dem Os lunatum über das semizirkuläre SL-Band (SL: scapholunär) verbunden, dessen stabilste Fasern (2-3× höhere Reißfestigkeit) auf der Dorsalseite liegen. Dies ist begründet in der Position des Skaphoids mit palmodistaler Lage des Tubercu-

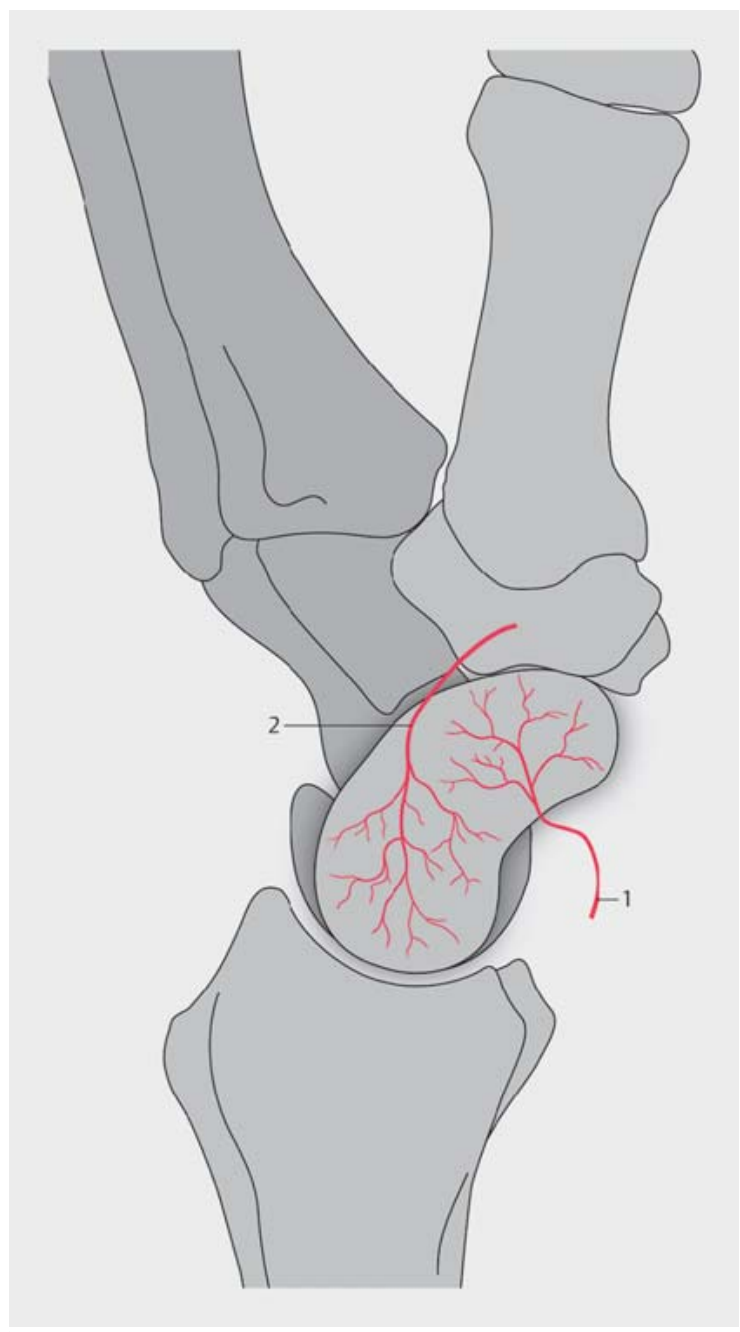

Abb. 1 Arterielle Versorgung des Skaphoids in der Seitenansicht. Ein palmarer (1) und ein dorsaler (2) Ast aus der A. radialis treten am distalen Pol in das Kahnbein ein, um sich nach proximal hin zu verzweigen. Der distale Skaphoidabschnitt ist von einem dichten Gefäßnetz durchzogen, während die intraossäre Gefäßdichte nach proximal geringer wird. Quelle: Mehling IM, Sauerbier M. Skaphoidfrakturen und Skaphoidpseudarthrosen. Z Orthop Unfall 2013; 151: 639-660. 


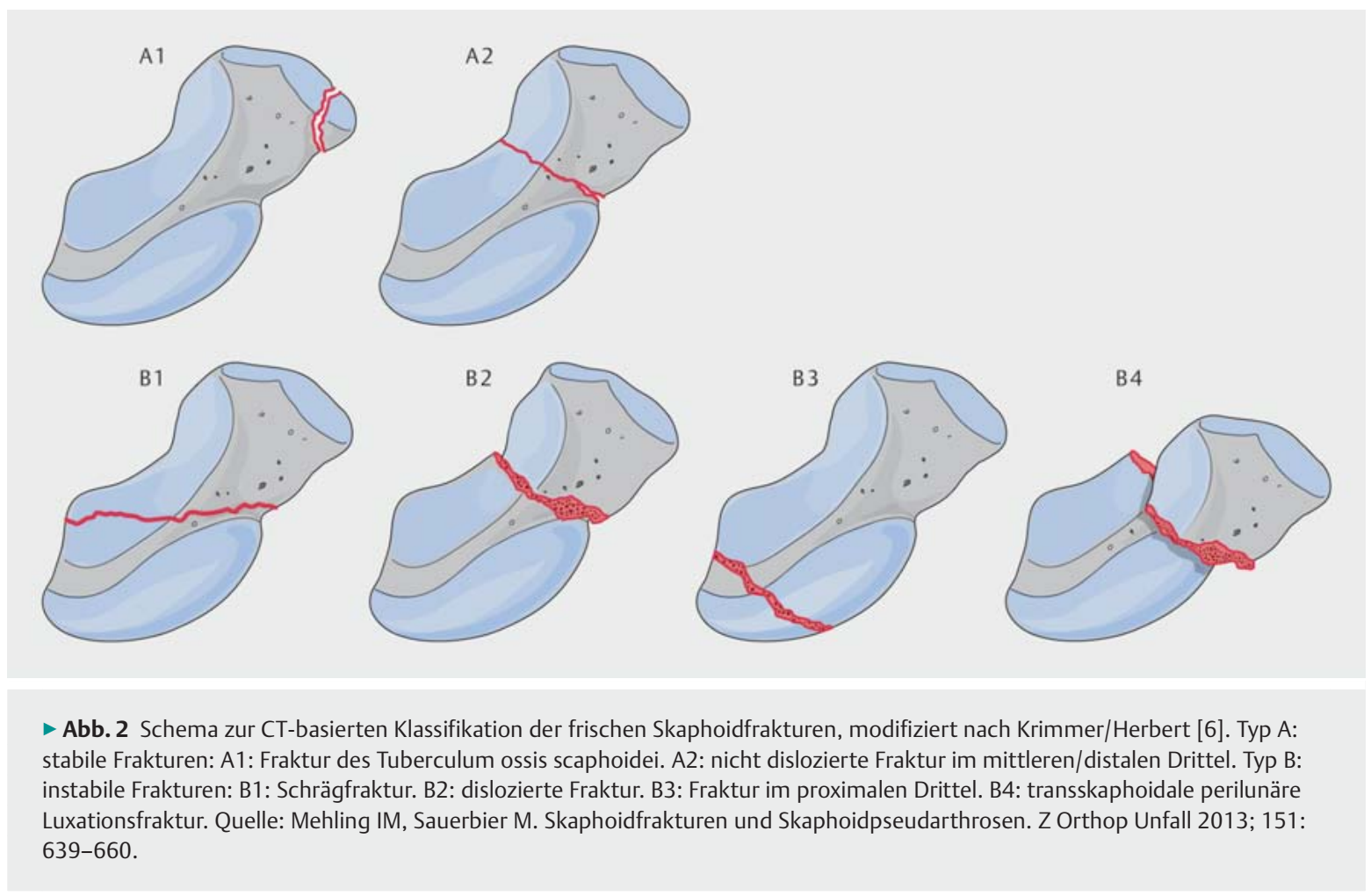

lum scaphoidei, das den Druck von Os trapezium und Os trapezoideum aufnehmen muss und somit das Kahnbein in eine Palmarflexion drängt. Gegen diese Kraft wirkt maßgeblich die Verspannung des Kahnbeins gegen das Os lunatum und das damit verbundene Os triquetrum, die wiederum eine Tendenz zur Dorsalflexion aufweisen [4].

\section{Frakturen}

Ungünstigerweise sind Brüche des Kahnbeins oft zunächst mit nur moderaten Beschwerden assoziiert und werden daher gar nicht oder nur verspätet diagnostiziert. Der konventionellen Röntgenbildgebung „entgeht“ in den Standardprojektionen dorsopalmar, lateral und „nach Stecher“ mit bis zu 30\% ein relevanter Anteil der Verletzungen, sodass bei entsprechenden klinischen Beschwerden eine Schnittbildgebung (a.e. CT) durchgeführt werden sollte [5].

Die CT des Handgelenkes sollte in Ausrichtung der Längsachse des Skaphoids - also in ca. $45^{\circ}$ zur Körperachse mit niedriger Schichtdicke (max. $1 \mathrm{~mm}$ ) durchgeführt werden, um auch kleinere Dislokationen sicher zu identifizieren.

Skaphoidfrakturen werden anhand der CT-Bildgebung nach Krimmer und Herbert in stabile Typ-A1- und -A2Frakturen sowie instabile Typ-B1-4-Frakturen eingeteilt [6] (॰ Abb. 2).
Eine konservative Therapie kommt generell nur für stabile Frakturen der Typen A1 (distale Tuberkelfrakturen) und A2 (undislozierte Frakturen mit querem Verlauf im mittleren und distalen Drittel) in Betracht. Hier wird allgemein eine Ruhigstellung im Unterarmgips mit Daumeneinschluss empfohlen, wobei in der Literatur die Empfehlungen zur Tragedauer des Gipses (von 4 bis über 12 Wochen) als auch die Form des Gipses (mit vs. ohne Daumeneinschluss) diskutiert werden [7]. Allerdings kann auch in diesen Fällen eine konservative Therapie nicht unüberlegt empfohlen werden, da Pseudarthroseraten von 1,5 bis zu $37 \%$ nach konservativer Therapie beschrieben sind [8]. Bei stabilen A2-Frakturen kann dem Patienten die operative Therapie ebenfalls angeboten werden, da den möglichen Komplikationen einer Operation die frühe funktionelle Mobilisation bereits nach kurzer Zeit entgegensteht.

Die instabilen Typ-B-Frakturen sollten operiert werden ( Abb. 3). B1-Frakturen (lange Schrägfrakturen) haben eine relativ hohe Kontaktfläche, wobei hier sekundäre Dislokationen und ein Abrutschen beobachtet werden. Dislozierte B2-Frakturen weisen häufig einen dorsalen „hump“ auf, der durch die Extensionstendenz des proximalen Fragmentes und die Flexionstendenz des distalen Fragmentes entsteht (s.o.). Frakturen des proximalen Pols (Typ B3) weisen die höchste Pseudarthroserate auf, da diese gehäuft eine Durchblutungsstörung zeigen, da keine Gefäße in den proximalen Pol direkt eintreten. Trotz operativer Stabilisierung findet sich eine Gesamt- 


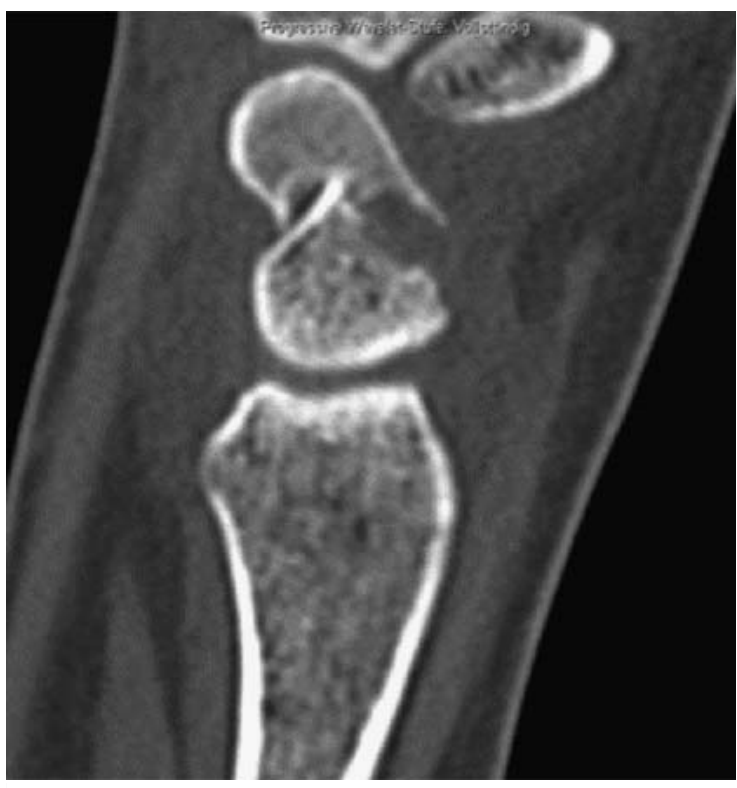

- Abb. 3 Instabile Fraktur mit „hump-back-Deformität“ und hohem Pseudarthroserisiko. Starke Dislokation mit geringem Fragmentkontakt.

pseudarthroserate von über 10\% [1]. Typ-B4-Frakturen (transskaphoidale perilunäre Luxationsfrakturen) stellen einen Sonderfall dar und müssen bei komplexer Instabilität des Karpus immer entsprechend operativ adressiert werden.

\section{Pseudarthrose}

Die normale, ungestörte Heilung des Skaphoids darf bis zu 12 Wochen dauern, bis eine knöcherne Konsolidierung nachweisbar sein sollte. Nach dieser Zeit spricht man von einer verzögerten Knochenheilung, „delayed union“. Von einer Scaphoidpseudarthrose spricht man bei ausbleibender knöcherner Konsolidierung nach 6 Monaten.

Es müssen 2 Arten von Pseudarthrosen unterschieden werden:

- „straffe“ Pseudarthrosen, bei denen die Fragmente mit Bindegewebe verbunden bleiben und eine geringe Dislokation im Verlauf aufweisen, und

- instabile Pseudarthrosen ohne bindegewebige Brücke und mit hoher Dislokationsrate.

Pseudarthrosen entstehen meist durch die Zusammenwirkung mehrerer Faktoren: starke Bewegung zwischen den Fragmenten verhindert die stabile Bildung von Kollagenbrücken und Knochenbälkchen, sodass die primär nicht erkannte Fraktur ohne entsprechende Therapie (Ruhigstellung oder OP) wohl das größte Risiko zur Entstehung eines Falschgelenkes darstellt. Ein geringer Fragmentkontakt, der bei einer „hump-back-Deformität“ oder ähnlich gelagerter Dislokation vorliegt sowie eine unzureichende Durchblutung, wie sie bei der proximalen Polfraktur besteht, führen ebenfalls zur unzureichenden Knochenheilung.

Durch die biomechanisch bedeutende Rolle des Skaphoids im karpalen Gefüge führt eine dauerhaft instabile Situation unweigerlich zur Änderung der Kinematik und somit zur Änderung der Belastung der Knorpelflächen der Handwurzel. Ein Abkippen des distalen Pols kann zu radioskaphoidalem Impingement mit arthrotischen Veränderungen in diesem Bereich führen. Eine Anschlussinstabilität kann - ähnlich einer SL-Band-Ruptur - im Laufe der Zeit eine DISI-Fehlstellung (DISI: Dorsiflexed intercalated Segment Instability) des Os lunatum nach sich ziehen und so zum karpalen Kollaps führen. Man spricht dann von einem SNAC Wrist (SNAC: Scaphoid Nonunion advanced Collapse) mit zunächst radioskaphoidaler und in späteren Stadien auch midkarpaler Arthrose $[9,10]$.

Filan und Herbert haben bereits 1996 die Klassifikation der Skaphoidfrakturen um eine Einteilung der Pseudarthrosen erweitert, die diesen Faktoren Rechnung trägt [11].

Die verzögerten Knochenheilungen (Typ C) mit nur geringer oder fehlender Dislokation haben das Potenzial, trotz erkennbarer Resorptionszone im weiteren Verlauf zu konsolidieren. Hier ist sowohl eine suffiziente Ruhigstellung (Unterarmgips mit Daumeneinschluss) als auch eine minimalinvasive Schraubenosteosynthese mit dem Patienten zu diskutieren, wobei Letztere zu favorisieren ist.

Die echten Pseudarthrosen des Typs D1-4 sollten in aller Regel einer operativen Therapie zugeführt werden. Hier kommen je nach Form der Pseudarthrose verschiedene Optionen in Betracht in Abhängigkeit von der Stellung der Fragmente und der Vitalität vor allem des proximalen Frakturanteils ( $\triangleright$ Abb. 4 ).

Zur exakten Diagnosestellung muss hier in jedem Fall wie auch bei der Skaphoidfraktur - eine Schnittbildgebung gefordert werden. Die CT-Diagnostik muss eine geringe Schichtdicke (unter $1 \mathrm{~mm}$ ) aufweisen sowie parallel zur Skaphoidachse im CT gelagert werden, um suffizient beurteilbare Bilder in multiplanaren Rekonstruktionen zu produzieren. Die MRT mit Kontrastmittel empfiehlt sich zur Vitalitätsabschätzung des proximalen Pols. Eine deutliche Mehrsklerosierung in der konventionellen Bildgebung oder der CT kann hier ein erster Hinweis auf eine avaskuläre Nekrose sein.

\section{Operative Therapie}

Die Therapie der Skaphoidverletzungen war bis Mitte der 1980er-Jahre eine Domäne der konservativen Therapie [8]. Osteosynthesen mit Schrauben (Streli-Navikulareschraube) oder mit Ender-Platte waren eher selten. Mit der Entwicklung der „Herbert-Schraube“ begann 1984 


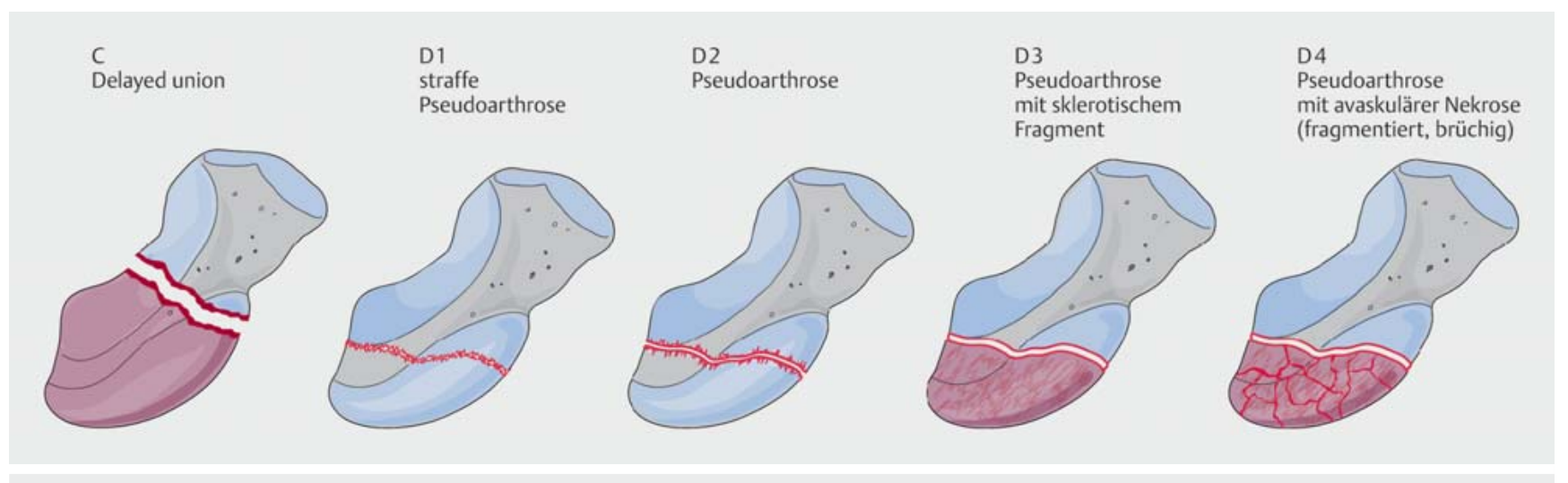

- Abb. 4 Klassifikation der Skaphoidpseudarthrose. Typ C: Resorptionsband an beiden Fragmenten parallel zum Frakturspalt. Typ D: D1: Straffe Pseudarthrose mit bindegewebiger Überbrückung, kein karpaler Kollaps. D2: Pseudarthrose mit instabilen Fragmenten und beginnender Deformität. D3: Sklerosierung entlang des Pseudarthrosespalts und zunehmender Deformität. D4: Pseudarthrose mit avaskulärem proximalem Fragment und karpaler Deformität. Quelle: Mehling IM, Sauerbier M. Skaphoidfrakturen und Skaphoidpseudarthrosen. Z Orthop Unfall 2013; 151: 639-660.

eine neue Ära der Versorgung dieser Verletzungen. Heute sind von diversen Herstellern teils selbst bohrende und selbst schneidende, subchondral zu liegen kommende, i.d.R. kanülierte Kompressionsschrauben verfügbar, mit der die meisten Skaphoidfrakturen adressiert werden können. Für multifragmentäre hoch instabile Situationen und besondere Pathologien ist ebenfalls eine palmare winkelstabile Plattenosteosynthese verfügbar (APTUS ${ }^{\circledR}$ Hand TriLock 1.5 Scaphoid Plate - Firma Medartis AG, Basel, Schweiz).

Definitionsgemäß gering dislozierte straffe D1-Pseudarthrosen können meist minimalinvasiv therapiert werden. Sowohl eine antegrade Verschraubung von dorsal als auch eine retrograde Schraubenosteosynthese von palmar sind möglich, wobei die Autoren nur bei eher proximalen Frakturen den dorsalen Zugang favorisieren.

Instabile, dislozierte, aber vitale D2- und D3-Pseudarthrosen erfordern meist eine offene oder arthroskopische Reposition sowie eine Spongiosaplastik, um die Wahrscheinlichkeit der Konsolidierung zu erhöhen.

Pseudarthrosen im mittleren Drittel und distale Pseudarthrosen (Letztere sind sehr selten) werden meist über einen palmaren Zugang versorgt. Der Zugang verläuft entlang der FCR-Sehne (FCR: Flexor Carpi Radialis) unter Schonung der A. radialis. Zur Kontrolle der Fragmente und zur Reposition können frei eingebrachte K-Drähte als „Joysticks“ genutzt werden. Die Ränder der Pseudarthrose müssen angefrischt und die Sklerosezone ( $\triangleright$ Abb. 5) reseziert werden, bis spongiöser Knochen allseitig vorhanden ist.

Dies ist ein sehr wichtiger Operationsschritt: Auf beiden Seiten müssen Spongiosaanteile gut sichtbar werden. Um die Flexionsstellung - den Hump („hump-backDeformität“) - zu korrigieren wird im offenen Verfahren

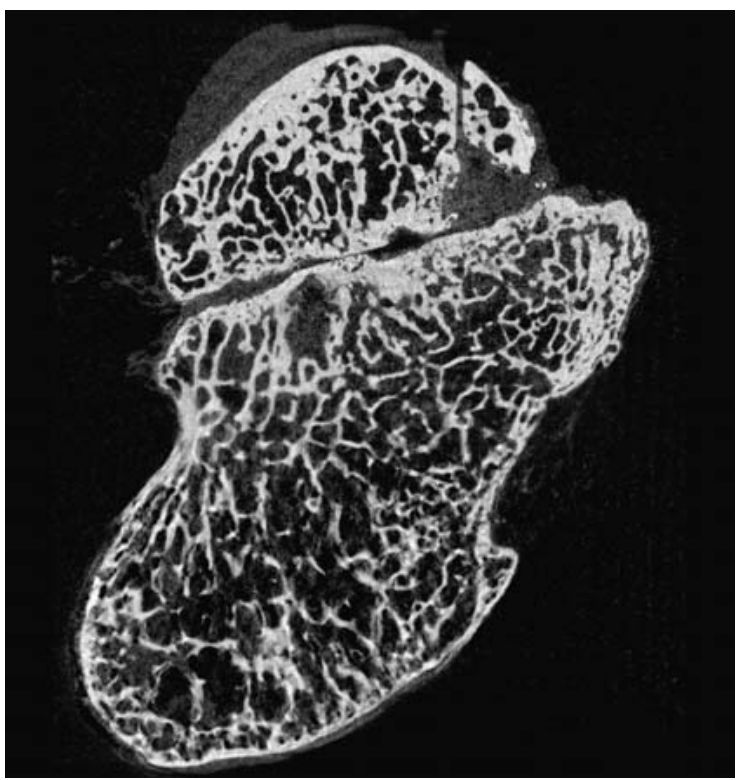

- Abb. 5 Mikro-CT einer Skaphoidpseudarthrose, beachte die Sklerose am ehem. Frakturrand.

ein Knochenspan vom distalen Radius oder dem Beckenkamm interponiert (erweiterte Matti-Russe-Plastik [12, 13] ( $\triangleright$ Abb. 6). Hierbei ist die Rekonstruktion der anatomischen Form des Skaphoids zur langfristigen Verhinderung einer Arthrose essenziell [14].

Die ursprünglich beschriebene Technik empfahl bei Bedarf eine K-Draht-Fixation des Transplantates - heute wird der Knochenspan in den meisten Fällen mit einer oder 2 Kompressionsschrauben, K-Drähten oder einer palmaren Platte befestigt. Eine erhöhte Konsolidierungsrate mit Osteosynthese ist nachgewiesen, eine Überlegenheit eines der Verfahren gegenüber einem anderen ist jedoch nicht nachweisbar. Insgesamt können mit die- 


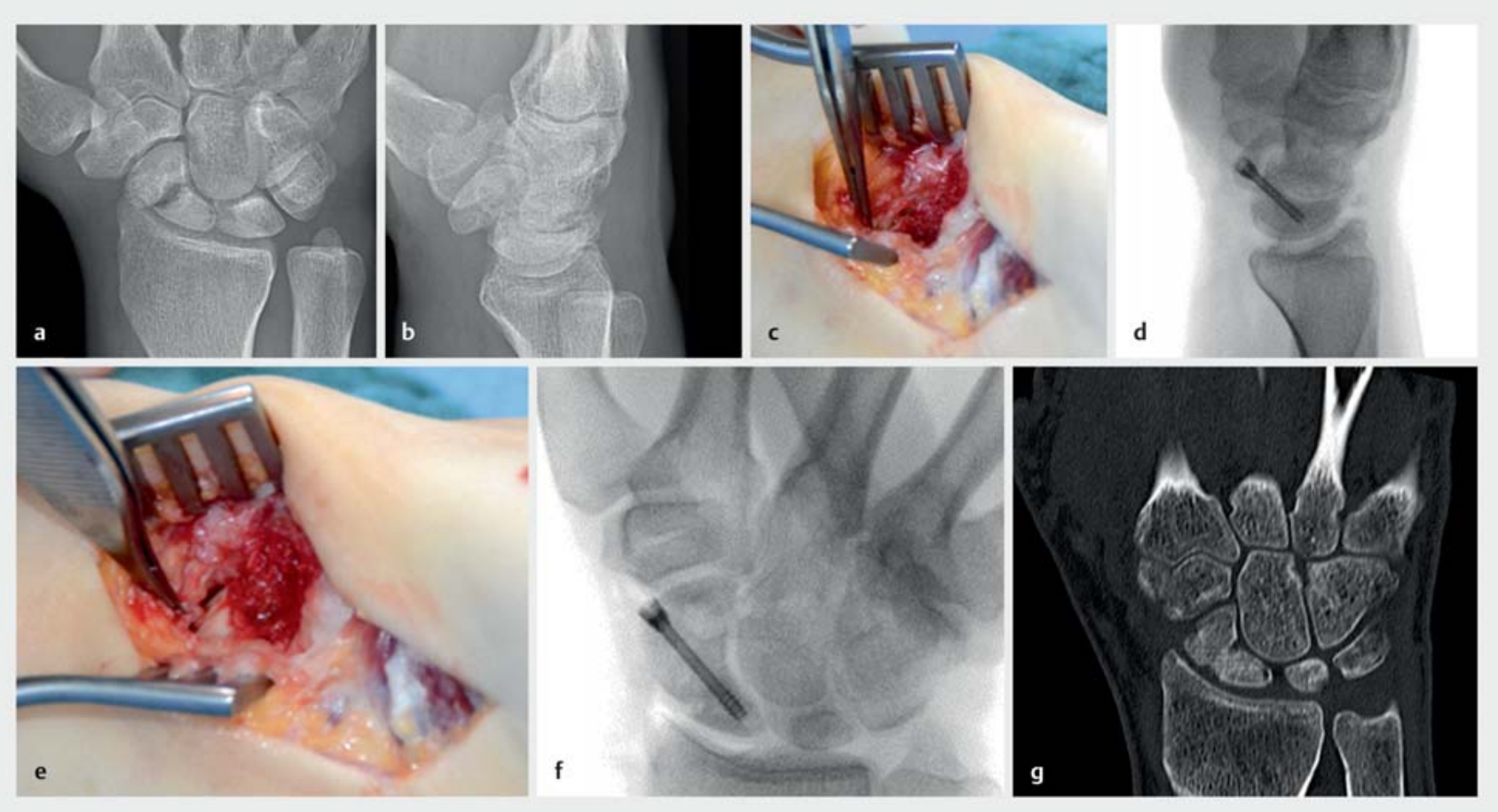

- Abb. 6 22-jähriger Patient ohne erinnerliches Trauma. Intraoperative Befunde: Resektion der Pseudarthrose, bis spongiöser Knochen vorliegt, Schraubenosteosynthese mit Beckenkammspan. Partielle Konsolidierung nach 21ำ Monaten (Bild der Konsolidierung neben der Schraube).

ser Technik Ausheilungsraten von 60 bis über $90 \%$ erreicht werden $[15,16]$.

Wichtig für eine Heilung der Pseudarthrose des Kahnbeins sind also 2 Dinge:

1. gute Knochenqualität auf beiden Seiten mit gutem interponierten Knochenspan und

2. gute Stabilität der Osteosynthese.

Proximale Pseudarthrosen sind über einen dorsalen Zugang gut zu visualisieren. Hierzu erfolgt die Inzision im Verlauf der EPL-Sehne (EPL: Extensor Pollicis Longus) über dem dorsalen Handgelenk und der Zugang über das 3. Strecksehnenfach. Die Technik entspricht dem beschriebenen palmaren Vorgehen, wobei zum einen besonders die dorsale Gefäßversorgung beachtet und geschont werden muss und zum anderen hier nur eine Stabilisierung mittels Schrauben oder Drähten möglich ist.

Durch die Fortentwicklung moderner arthroskopischer Techniken ist erfahrenen Operateuren auch eine geschlossene Aufrichtung und Pseudarthrosenresektion mit arthroskopischer Spongiosaplastik möglich. Auch hier sind Ausheilungsraten von über $90 \%$ beschrieben, wobei die Verbreitung dieser Technik insgesamt als eingeschränkt betrachtet werden muss und sicher nicht bei jedem Handchirurgen ein solches Ergebnis erwartbar sein wird [17].
Pseudarthrosen mit avitalem proximalem Anteil Typ D4 meist isolierte Polfragmente unterschiedlicher Größe können zur Erhöhung der Ausheilungsrate mit einem vaskularisierten Span versorgt werden, um frisches durchblutetes Gewebe in das Scaphoid zu bringen. Hierzu besteht die Option einer lokal gestielten Versorgung mit einem Knochenspan vom distalen Radius. Der distale Radius wird dorsalseitig von mehreren interkompartimentellen supraretinakulären retrograd verlaufenden Arterien versorgt - hier bestehen Hebemöglichkeiten an der 1./2., 2./3. und 4./5. interkompartimentellen Arterie [18], wobei eben diese anatomische Gegebenheit auch die Ausdehnung und Positionierung des Transplantates bestimmen. Alternativ favorisieren einige Autoren freie gefäßgestielte Knochenblöcke zur Rekonstruktion des Skaphoids [19] ( A Abb. 7).

Hier zeigen gefäßgestielte Blöcke aus dem Beckenkamm gute Ergebnisse. In den letzten Jahren ist die Transposition eines gefäßgestielten medialen Femurspanes in den Fokus einiger Zentren gerückt. Die Versorgung erfolgt über eine relativ großlumige $A$. genicularis descendens, wodurch ein verhältnismäßig großer Block - bei Bedarf auch mit Indikatorhautinsel - gehoben werden kann. Hiermit können einigen Autoren zufolge höhere und schnellere Ausheilungsraten erzielt werden [19-22]. Die Hebemorbidität sowohl am Beckenkamm als auch am 


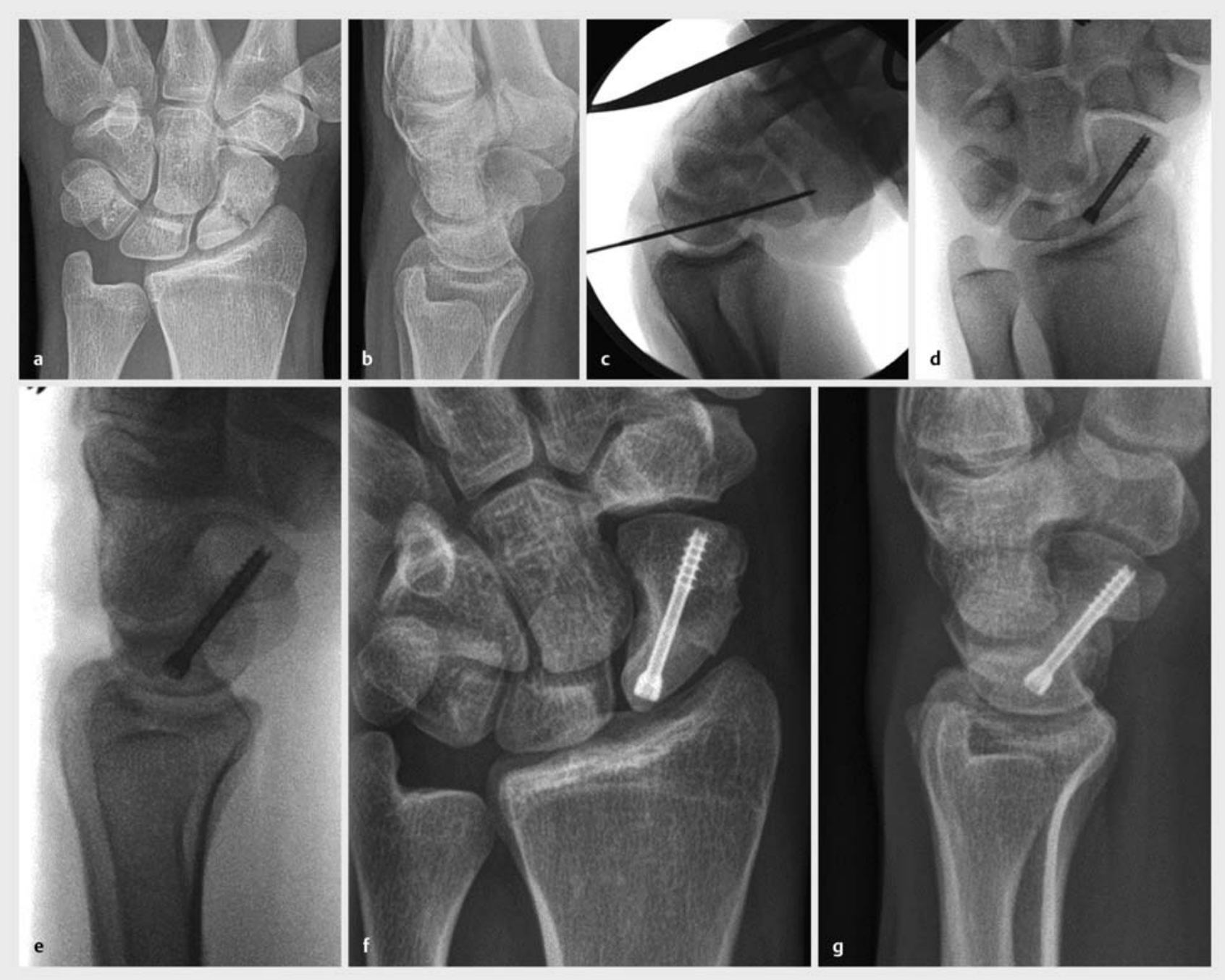

- Abb. 7 20-jähriger Patient nach Skiunfall. a,b 9 Monate nach Trauma. c-e ORIF-CCS-Schraube (Fa Medartis), Spongiosatransplantation vom ipsilateralen Radius. f,g Verlauf nach 1a, Range of Motion frei.

distalen Femur und die perioperativen Risiken sind hier jedoch nicht zu vernachlässigen.

Bei Revisionsoperationen wird von einer Vielzahl der Autoren die Verwendung eines vaskularisierten Spanes favorisiert, um zu den mechanischen Voraussetzungen einer knöchernen Heilung noch ein biologisches Additiv hinzuzubringen [23]. Auch eine extrakorporale Stoßwellentherapie (ESWT) scheint Vorteile in Bezug auf die Knochenheilung zu bringen - hier ist die Datenlage aus unserer Sicht aktuell noch nicht valide genug, um einen definitiven Vorteil zu beweisen [16]. Voraussetzung hierfür ist eine stabile Osteosynthese ohne Lockerungszeichen des Osteosynthesematerials.

Dem gegenüber stehen die Risikofaktoren einer persistierenden Pseudarthrose. Hier muss sicherlich neben einem nicht optimalen Operationsergebnis mit instabiler Ver- sorgung und nicht anatomischer Rekonstruktion des Skaphoids der chronische Nikotinkonsum als größter Risikofaktor genannt werden [24]. Weitere Risikofaktoren sind schwere körperliche Arbeit, Persistenz der Pseudarthrose über 5 Jahre, Radiusstyloidektomie und Dauer der Ruhigstellung post OP [25].

\section{Fazit}

Skaphoidpseudarthrosen treten nach Skaphoidfrakturen in über $30 \%$ der Fälle auf - auch nach operativer Therapie heilen ca. 10\% der Frakturen nicht entsprechend aus. Hieraus resultiert im schlechtesten Fall eine progrediente Arthrose des Handgelenks mit konsekutiven Bewegungseinschränkungen und Schmerzen. Die Therapieoptionen reichen von der minimalinvasiven Verschraubung straffer, gut stehender Pseudarthrosen bis zu hoch komplexen Rekonstruktionen des Skaphoids mit freien mikrovaskulär 
anastomosierten Knochenspänen bei avaskulären Nekrosen mit Strukturstörungen des Knochens. Allen Therapien muss eine Bildgebung mit Dünnschicht-CT mit entsprechenden Rekonstruktionen und ggf. kontrastmittelgestützem MRT vorausgehen, um die optimale Therapie präzise zu planen.

\section{Autorinnen/Autoren}

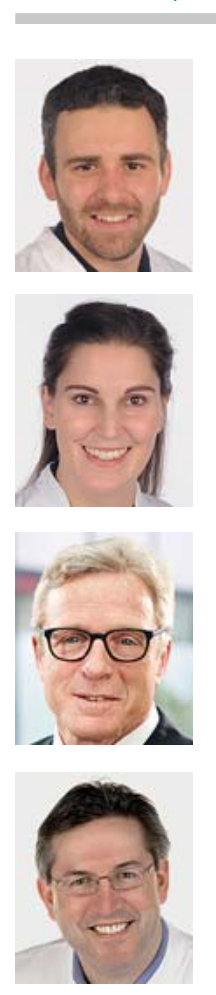

\section{Simon Oeckenpöhler}

Dr. med., Funktionsoberarzt der Klinik für Unfall-, Hand- und Wiederherstellungschirurgie, Universitätsklinikum Münster, Deutschland

\section{Johanna Ueberberg}

Assistenzärztin der Klinik für Unfall-, Handund Wiederherstellungschirurgie, Universitätsklinikum Münster, Deutschland

\section{Michael J. Raschke}

Univ.-Prof. Dr. med., Direktor der Klinik für Unfall-, Hand- und Wiederherstellungschirurgie, Universitätsklinikum Münster, Deutschland

\section{Martin F. Langer}

Prof. Dr. med., Sektionsleiter Handchirurgie der Klinik für Unfall-, Hand- und Wiederherstellungschirurgie, Universitätsklinikum Münster, Deutschland

\section{Korrespondenzadresse}

\section{Dr. med. S. Oeckenpöhler}

Klinik für Unfall-, Hand- und Wiederherstellungschirurgie Universitätsklinikum Münster

Waldeyerstraße 1

48149 Münster

Simon.Oeckenpoehler@ukmuenster.de

\section{Literatur}

[1] Dy C], Kazmers NH, Baty J et al. An epidemiologic perspective on scaphoid fracture treatment and frequency of nonunion surgery in the USA. HSS J 2018; 14: 245-250. doi:10.1007/ s11420-018-9619-3

[2] Botte M], Mortensen WW, Gelberman RH et al. Internal vascularity of the scaphoid in cadavers after insertion of the Herbert screw. J Hand Surg Am 1988; 13: 216-220

[3] Langer MF, Unglaub F, Breiter $S$ et al. Anatomie und Pathobiomechanik des Skaphoids. Unfallchirurg 2019; 122: 170-181. doi:10.1007/s00113-018-0597-1

[4] Langer MF, Oeckenpöhler S, Breiter S et al. Anatomie und Biomechanik des Kahnbeins. Orthopäde 2016; 45: 926-937. doi:10.1007/s00132-016-3339-5

[5] Schmitt R, Rosenthal H, Deutsche Gesellschaft für Unfallchirurgie et al. Bildgebende Diagnostik der Skaphoidfrakturen nach den aktuellen S3-Leitlinien. Fortschr Röntgenstr 2016; 188: 459-469. doi:10.1055/s-0042-104660

[6] Krimmer H, Schmitt R, Herbert T. Kahnbeinfrakturen - Diagnostik, Klassifikation und Therapie. Unfallchirurg 2000; 103: 812-819

[7] Schädel-Höpfner M, Bickert B, Dumont C et al. Die frische Skaphoidfraktur Management unter Berücksichtigung der neuen S3-Leitlinie. Orthopäde 2016; 45: 945-950. doi:10.1007/ s00132-016-3336-8

[8] Baumeister HH, Greinemann H. Zur konservativen Behandlung des Skaphoidbruches der Handwurzel. Unfallchirurg 1989; 92: 175-179

[9] Mehling IM, Sauerbier M. Skaphoidfrakturen und Skaphoidpseudarthrosen. Z Orthop Unfall 2013; 151: 639-660. doi:10.1055/s-0033-1360131

[10] Mack GR, Bosse MJ, Gelberman RH et al. The natural history of scaphoid non-union. J Bone Joint Surg Am 1984; 66: 504-509

[11] Filan SL, Herbert TJ. Herbert screw fixation of scaphoid fractures. J Bone Joint Surg Br 1996; 78: 519-529

[12] Matti H. Technik und Resultate meiner Pseudarthrosenoperation. Zentralbl Chir 1936; 63: 11

[13] Russe O. Behandlungsergebnisse der Spongiosaauffüllung bei Kahnbeinpseudarthrosen. Z Orthop Ihre Grenzgeb 1951; 81: 466-473

[14] Daecke W, Wieloch P, Vergetis P et al. Occurrence of carpal osteoarthritis after treatment of scaphoid nonunion with bone graft and Herbert screw: a long-term follow-up study. J Hand Surg Am 2005; 30: 923-931. doi:10.1016/j.jhsa.2005.05.017

[15] Pinder RM, Brkljac M, Rix L et al. Treatment of Scaphoid Nonunion: A Systematic Review of the Existing Evidence. J Hand Surg Am 2015; 40: 1797-1805.e3. doi:10.1016/j.jhsa.2015.05.003

[16] Quadlbauer S, Pezzei C, Beer T et al. Treatment of scaphoid waist nonunion by one, two headless compression screws or plate with or without additional extracorporeal shockwave therapy. Arch Orthop Trauma Surg 2019; 139: 281-293. doi:10.1007/s00402-018-3087-6

[17] Wong WY, Ho PC. Minimal invasive management of scaphoid fractures: from fresh to nonunion. Hand Clin 2011; 27: 291307. doi:10.1016/j.hcl.2011.06.003

[18] Zaidenberg C, Siebert JW, Angrigiani C. A new vascularized bone graft for scaphoid nonunion. J Hand Surg Am 1991; 16: 474-478

[19] Tsantes AG, Papadopoulos DV, Gelalis ID et al. The efficacy of vascularized bone grafts in the treatment of scaphoid nonunions and Kienbock disease: a systematic review in 917 patients. J Hand Microsurg 2019; 11: 6-13. doi:10.1055/s0038-1677318

[20] Hertel R, Masquelet AC. The reverse flow medial knee osteoperiosteal flap for skeletal reconstruction of the leg. Description and anatomical basis. Surg Radiol Anat 1989; 11: 257262

[21] Jones DB, Jr., Burger H, Bishop AT et al. Treatment of scaphoid waist nonunions with an avascular proximal pole and carpal collapse. A comparison of two vascularized bone grafts. J Bone Joint Surg Am 2008; 90: 2616-2625. doi:10.2106/JBJS. G.01503

[22] Kazmers NH, Thibaudeau S, Levin LS. A scapholunate ligament-sparing technique utilizing the medial femoral condyle corticocancellous free flap to reconstruct scaphoid nonunions with proximal pole avascular necrosis. J Hand Surg Am 2016; 41: e309-e315. doi:10.1016/j.jhsa.2016.06.004 
[23] Wolfe S, Pederson W, Kozin SH, Cohen M, eds. Green's operative Hand Surgery. 7th ed. Philadelphia: Elsevier; 2017

[24] Dinah AF, Vickers RH. Smoking increases failure rate of operation for established non-union of the scaphoid bone. Int Orthop 2007; 31: 503-505. doi:10.1007/s00264-006-0231-7

[25] Schuind F, Haentjens P, Van Innis F et al. Prognostic factors in the treatment of carpal scaphoid nonunions. J Hand Surg Am 1999; 24: 761-776
Bibliografie

DOI https://doi.org/10.1055/a-0898-9321

OP-JOURNAL 2019; 35: 292-299 @ Georg Thieme Verlag KG Stuttgart · New York ISSN 0178-1715 